Article

\title{
Existence and Uniqueness of Solutions for the $p(x)$-Laplacian Equation with Convection Term
}

\author{
Bin-Sheng Wang ${ }^{1}$, Gang-Ling Hou ${ }^{1}$ and Bin Ge ${ }^{2, *(D)}$ \\ 1 College of Aerospace and Civil Engineering, Harbin Engineering University, Harbin 150001, China; \\ wangbinsheng@hrbeu.edu.cn (B.-S.W.); hougl@hrbeu.edu.cn (G.-L.H.) \\ 2 College of Mathematical Sciences, Harbin Engineering University, Harbin 150001, China \\ * Correspondence: gebin791025@hrbeu.edu.cn
}

Received: 7 September 2020; Accepted: 9 October 2020; Published: 13 October 2020 updates

\begin{abstract}
In this paper, we consider the existence and uniqueness of solutions for a quasilinear elliptic equation with a variable exponent and a reaction term depending on the gradient. Based on the surjectivity result for pseudomonotone operators, we prove the existence of at least one weak solution of such a problem. Furthermore, we obtain the uniqueness of the solution for the above problem under some considerations. Our results generalize and improve the existing results.
\end{abstract}

Keywords: $p(x)$-Laplacian equation; convection term; pseudomonotone operators; existence results; uniqueness

\section{Introduction and Main Results}

The $p(x)$-Laplacian operator has been used in the modelling of electrorheological fluids ([1-3]), in elastic mechanics ([4]), in image restoration ([5-7]) and in magnetostatics problems ([8]). Up to now, a large number of results have been obtained for solutions to equations related to this operator. For instance, we must mention the works of Kefi-Radulescu [9], Saoudi [10], Kefi-Saoudi [11], Xie-Chen [12], Nhan-Chuong-Truong [13], Zhang [14], Zhang-Motreanu [15], Yucedag [16], Yin-Li-Ke [17], Ait Hammou-Azroul-Lahmi [18], Ayazoglu-Ekincioglu [19], Ge-Wang [20], Ge-Lv [21], Ge-Radulescu [22], Heidarkhani-Afrouzi-Moradi-Caristi-Ge [23], Ge-Zhou-Wu [24], Fan [25] and the references therein. In relation to the regularity for solutions of differential equations with $p(x)$-Laplacian operator, we refer the readers to to [26-36], respectively, and the references therein. In this paper, we consider the following problem

$$
\begin{cases}-\operatorname{div}\left(|\nabla u|^{p(x)-2} \nabla u\right)=f(x, u, \nabla u), & \text { in } \Omega, \\ u=0, & \text { on } \partial \Omega\end{cases}
$$

where $\Omega$ is a smooth bounded domain in $\mathbb{R}^{N}, N \geq 2, f: \Omega \times \mathbb{R} \times \mathbb{R}^{N} \mapsto \mathbb{R}$ is a Carathéodory function, $p(\cdot)$ is a logarithmic Hölder continuous with values in $(1, N)$ and satisfies the following condition:

$\left(H_{p}\right)$ there is a vector $\xi_{0} \in \mathbb{R}^{N} \backslash\{0\}$ such that for any $x \in \Omega$, the map $t \mapsto p\left(x+t \tau_{0}\right)$ is monotone on $t \in\left\{t \mid x+t \xi_{0} \in \Omega\right\}$.

If $p(x) \equiv p$ is a constant, the equation in the problem (1) is of the form

$$
\begin{cases}-\operatorname{div}\left(|\nabla u|^{p-2} \nabla u\right)=f(x, u, \nabla u), & \text { in } \Omega, \\ u=0, & \text { on } \partial \Omega .\end{cases}
$$

Problems of form (2) arise naturally as stationary states of certain models in fluid mechanics. Therefore, it is important to obtain information about the existence and nonexistence of solutions for this problem. 
There is a rich body of literature concerning problem (2), see, e.g., [37-41] and the references therein. Using topological methods, Montenegro [37] and Ruiz [38] obtained the existence of positive solutions for problem (2), respectively. Later, Zou [39] proved that (2) has a positive solution by using the fixed point theorem with the a priori estimates. Very recently, the authors in [41] used sub-supersolution techniques with Schaefer's fixed point theorem to prove the existence of a positive and a negative solution for problem (2).

The key research point in the paper is that the right-hand side of problem (1) depends on the solution $u$ and its gradient $\nabla u$. The expression $f(x, u, \nabla u)$ is often referred to as a convection term. Since the nonlinearity $f$ depends on the gradient $\nabla u$, problem (1) does not have a variational structure, so the variational methods cannot be applied directly. In order to overcome this difficulty, Yin, Li and Ke in [17], by using Krasnoselskii's fixed point theorem on the cone, proved the existence of positive solutions for problem (1) under certain assumptions. Very recently, the authors in [18] used the topological degree theory for a class of demicontinuous operators of generalized $\left(S_{+}\right)$type to prove the existence of at least one weak solution for problem (1) under the following assumption on $f(x, t, \xi)$ :

$\left(H_{f}\right)$ There exists $k \in L^{\frac{p(x)}{p(x)-1}}(\Omega), q \in C(\Omega)$ with $1<q^{-} \leq q(x) \leq q^{+}<p^{-}$and $C>0$ such that

$$
|f(x, t, \xi)| \leq C\left(k(x)+|t|^{q(x)-1}+|\xi|^{q(x)-1}\right)
$$

for a.a. $x \in \Omega$ and all $(t, \xi) \in \mathbb{R} \times \mathbb{R}^{N}$.

It is well known that the condition $\left(H_{f}\right)$ means that the nonlinearity $f$ has sub- $p$ linear growth. However, this condition is quite restrictive and eliminates several nonlinearities. Motivated by the aforementioned works, in the present paper, we consider the problem (1) in the case when the nonlinearity $f$ satisfies the subcritical growth condition. To this end, we assume that $f: \Omega \times \mathbb{R} \times \mathbb{R}^{N} \mapsto$ $\mathbb{R}$ is a Carathéodory function that satisfies the following assumptions:

$\left(f_{1}\right)$ There exists $k \in L^{\frac{r(x)}{r(x)-1}}(\Omega), 1<r(x)<p^{*}(x):=\frac{N p(x)}{N-p(x)}$ and $C>0$ such that

$$
|f(x, t, \xi)| \leq C\left(k(x)+|t|^{r(x)-1}+|\xi|^{p(x) \frac{r(x)-1}{r(x)}}\right)
$$

for a.a. $x \in \Omega$ and all $(t, \xi) \in \mathbb{R} \times \mathbb{R}^{N}$.

$\left(f_{2}\right)$ There exists $w \in L^{1}(\Omega)$ and $b_{1}, b_{2} \geq 0$ with $b_{1}+b_{2} \lambda_{*}^{-1}<1$ such that

$$
f(x, t, \xi) t \leq b_{1}|\xi|^{p(x)}+b_{2}|t|^{p(x)}+w(x)
$$

for a.a. $x \in \Omega$ and all $(t, \xi) \in \mathbb{R} \times \mathbb{R}^{N}$, where $\lambda_{*}$ is the infimum of all eigenvalues of the nonhomogeneous eigenvalue problem

$$
\begin{cases}-\operatorname{div}\left(|\nabla u|^{p(x)-2} \nabla u\right)=\lambda|u|^{p(x)-2} u, & \text { in } \Omega, \\ u=0, & \text { on } \partial \Omega,\end{cases}
$$

Remark 1. It is well known that (see ([42] Theorem 3.4)), if condition $\left(H_{p}\right)$ holds, then we have $\lambda_{*}:=$ $\inf _{u \in W_{0}^{1, p(x)}(\Omega) \backslash\{0\}} \frac{\int_{\Omega}|\nabla u|^{p(x)} d x}{\int_{\Omega}|u|^{p(x)} d x}>0$.

Remark 2. The following function satisfies conditions $\left(f_{1}\right)-\left(f_{2}\right)$, where, for simplicity, we drop the $x$-dependence,

$$
\begin{aligned}
& \qquad f(t, \xi)=-c_{1}|t|^{r(x)-2} t+c_{2}|\xi|^{p(x)-1}, \forall t \in \mathbb{R}, \forall \xi \in \mathbb{R}^{N}, \\
& \text { with } p(x)<r(x)<p^{*}(x), c_{1} \geq 0 \text { and } 0 \leq c_{2}<\frac{1}{1+\lambda_{1, \alpha}^{-1}} .
\end{aligned}
$$


We are now in the position to state our main result.

Theorem 1. Assume that $\left(H_{p}\right)$ and $\left(f_{1}\right)-\left(f_{2}\right)$ hold. Then, problem (1) has at least one solution.

Next, we consider the uniqueness of solutions of (1). To this end, we also need the following hypotheses on the convection term:

$\left(f_{3}\right)$ There exists $b_{3} \geq 0$ such that

$$
(f(x, t, \xi)-f(x, s, \xi))(t-s) \leq b_{3}|t-s|^{p(x)}
$$

for a.a. $x \in \Omega$ and all $(t, \xi) \in \mathbb{R} \times \mathbb{R}^{N}$.

$\left(f_{4}\right)$ There exists $b_{4} \geq 0$ such that

$$
|f(x, t, \xi)-f(x, t, \eta)| \leq b_{4}|\xi-\eta|^{p(x)-1}
$$

for a.a. $x \in \Omega$ and all $(t, \xi) \in \mathbb{R} \times \mathbb{R}^{N}$.

Our uniqueness result reads as follows.

Theorem 2. Assume that $\left(H_{p}\right)$ and $\left(f_{1}\right)-\left(f_{4}\right)$ hold. If, in addition, $2 \leq p(x)$ for all $x \in \bar{\Omega}$, then problem (1) has a unique weak solution provided

$$
2^{p^{+}}\left(\left[b_{3}+\frac{b_{4}}{q^{-}}\right] \lambda_{* *}^{-1}+b_{4} \frac{q^{+}-1}{q^{-}}\right)<1 .
$$

To the best of our knowledge, our setting is more general than those of $[17,18]$ and our method contrasts with other treatments of (1). The functional framework contains the generalized Lebesgue and Sobolev spaces with variable exponents and our technique is based on the surjectivity result for pseudomonotone operators.

This paper is designed as follows: First, we briefly review the definitions and collect some preliminary results for the variable exponent Sobolev spaces $W_{0}^{1, p(x)}(\Omega)$ and we recall the surjectivity result for pseudomonotone operators. Finally, we complete the proofs of Theorems 1 and 2 of this paper.

\section{Preliminaries}

In order to discuss problem (1), we start with the definition of the variable exponent Lebesgue spaces $L^{p(x)}(\Omega)$ and the the variable exponent Sobolev spaces $W_{0}^{1, p(x)}(\Omega)$, and some properties of them; for more details, see [43-46].

Let $\Omega$ and $p$ be as in Section 1. Let us denote

$$
C_{+}(\bar{\Omega})=\{h \in C(\bar{\Omega}): h(x)>1 \text { for every } x \in \bar{\Omega}\}
$$

For any $h \in C_{+}(\bar{\Omega})$, we write

$$
h^{-}:=\min _{x \in \bar{\Omega}} h(x) \text { and } h^{+}:=\max _{x \in \bar{\Omega}} h(x) .
$$

For any $p \in C_{+}(\bar{\Omega})$, we define the variable exponent Lebesgue space by

$$
L^{p(x)}(\Omega)=\left\{u \text { is a measurable real valued function with } \int_{\Omega}|u|^{p(x)} d x<+\infty\right\} .
$$

We consider this space to be endowed with the so-called Luxemburg norm:

$$
|u|_{p(x)}=\inf \left\{\lambda>0: \int_{\Omega}\left|\frac{u(x)}{\lambda}\right|^{p(x)} d x \leq 1\right\} .
$$


We define the variable exponent Sobolev spaces $W^{1, p(x)}(\Omega)$ by

$$
W^{1, p(x)}(\Omega)=\left\{u \in L^{p(x)}(\Omega):|\nabla u| \in L^{p(x)}(\Omega)\right\},
$$

equipped with the norm

$$
\|u\|=|u|_{p(x)}+|\nabla u|_{p(x)}
$$

The space $W_{0}^{1, p(x)}(\Omega)$ is defined by the closure of $C_{0}^{\infty}(\Omega)$ in $W^{1, p(x)}(\Omega)$. With these norms, the spaces $L^{p(x)}(\Omega), W_{0}^{1, p(x)}(\Omega)$ and $W^{1, p(x)}(\Omega)$ are separable reflexive Banach spaces; see [44,46] for details.

Proposition 1. ([44]) Set $\rho(u)=\int_{\Omega}|u|^{p(x)} d x$. Let $u \in L^{p(x)}(\Omega)$, then, we have

(i) $|u|_{p(x)}<1(=1 ;>1) \Leftrightarrow \rho(u)<1(=1 ;>1)$.

(ii) If $|u|_{p(x)} \geq 1$, then $|u|_{p(x)}^{p^{-}} \leq \rho(u) \leq|u|_{p(x)}^{p^{+}}$.

(iii) If $|u|_{p(x)} \leq 1$, then $|u|_{p(x)}^{p^{+}} \leq \rho(u) \leq|u|_{p(x)}^{p^{-}}$.

Proposition 2. $([44,46])$

(1) If $q \in C_{+}(\bar{\Omega})$ and $q(x)<p^{*}(x)$ for every $x \in \Omega$, then the embedding from $W_{0}^{1, p(x)}(\Omega)$ to $L^{q(x)}(\Omega)$ is continuous and compact.

(2) The Poincare's inequality in $W_{0}^{1, p(x)}(\Omega)$ holds, that is, there is a constant $C_{0}>0$ such that

$$
|u|_{p(x)} \leq C_{0}|\nabla u|_{p(x)}, \forall u \in W_{0}^{1, p(x)}(\Omega) .
$$

It follows from (1) of Proposition 2 that there exists $c_{\vartheta}>0$ such that

$$
|u|_{q(x)} \leq c_{\vartheta}\|u\|, \forall u \in W_{0}^{1, p(x)}(\Omega)
$$

We note that by (2) of Proposition 2, it follows that $|\nabla u|_{p(x)}$ is an equivalent norm in $W_{0}^{1, p(x)}(\Omega)$. We will use the equivalent norm in the following discussion and write $\|u\|=|\nabla u|_{p(x)}$ for simplicity.

Let us recall some definitions we will use later.

Definition 1. Let $X$ be a reflexive Banach space, $X^{*}$ its dual space and let $\langle\cdot, \cdot\rangle$ denote its duality pairing. Let $\mathcal{L}: X \rightarrow X^{*}$; then, $\mathcal{L}$ is called

(i) to satisfy the $\left(S_{+}\right)$-property if $u_{n} \rightarrow u$ in $X$ and $\limsup _{n \rightarrow+\infty}\left\langle\mathcal{L}\left(u_{n}\right), u_{n}-u\right\rangle \leq 0$ imply $u_{n} \rightarrow u$ in $X$;

(ii) pseudomonotone if $u_{n} \rightarrow u$ in $X$ and $\limsup _{n \rightarrow+\infty}\left\langle\mathcal{L}\left(u_{n}\right), u_{n}-u\right\rangle \leq 0$ imply $\mathcal{L}\left(u_{n}\right) \rightarrow \mathcal{L}(u)$ and $\left\langle\mathcal{L}\left(u_{n}\right), u_{n}\right\rangle \rightarrow\langle\mathcal{L}(u), u\rangle$.

The main tool is based on the surjectivity result for pseudomonotone operators from Carl-Le-Motreanu ([47] Theorem 2.99), which is now stated for the reader's convenience.

Lemma 1. Let $X$ be a real reflexive Banach space, and assume that $\mathcal{L}: X \rightarrow X^{*}$ is a bounded, coercive and pseudomonotone operator. Then, there exists a solution to the equation $\mathcal{L}(u)=v$, where $v \in X^{*}$.

Now, we denote the $p(x)$-Laplacian operator $\mathcal{L}: W_{0}^{1, p(x)}(\Omega) \rightarrow\left(W_{0}^{1, p(x)}(\Omega)\right)^{*}$ defined by

$$
\langle\mathcal{L}(u), v\rangle=\int_{\Omega}|\nabla u|^{p(x)-2} \nabla u \cdot \nabla v d x
$$


for all $u, v \in W_{0}^{1, p(x)}(\Omega)$, where $\left(W_{0}^{1, p(x)}(\Omega)\right)^{*}$ denotes the dual space of $W_{0}^{1, p(x)}(\Omega)$ and $\langle\cdot, \cdot\rangle$ denotes the pairing between $W_{0}^{1, p(x)}(\Omega)$ and $\left(W_{0}^{1, p(x)}(\Omega)\right)^{*}$. The properties of double phase operator $\mathcal{L}$ are summarized in the following proposition; see Fan-Zhang ([43] Theorem 3.1).

Proposition 3. Let $\mathcal{L}$ be as before and set $E=W_{0}^{1, p(x)}(\Omega)$. Then, $\mathcal{L}: E \rightarrow E^{*}$ is a continuous, bounded, strictly monotone operator of type $\left(S_{+}\right)$, i.e., if $u_{n} \rightarrow u$ in $E$ and $\lim \sup \left\langle\mathcal{L}\left(u_{n}\right)-\mathcal{L}(u), u_{n}-u\right\rangle \leq 0$, implies $u_{n} \rightarrow u$ in $E$.

\section{Proof of Theorem 1}

In this section, the proof of the existence of nontrivial weak solutions for (1) is provided by applying the Lemma 1 under suitable assumptions. Recall that $u$ is said to be a weak solution of (1) if

$$
\int_{\Omega}|\nabla u|^{p(x)-2} \nabla u \cdot \nabla v d x=\int_{\Omega} f(x, u, \nabla u) v d x
$$

is satisfied for all test functions $v \in E$. Taking Proposition 2(1) into account, along with the growth conditions in $\left(f_{1}\right)$, we see that the definition of a weak solution is well defined.

We now turn to the proof of Theorem 1.

Proof of Theorem 1. Define the Nemytskii operator $\bar{N}_{f}: E \subseteq L^{r(x)}(\Omega) \rightarrow L^{\frac{r(x)}{r(x)-1}}(\Omega)$ defined by

$$
\left(\bar{N}_{f} u\right)(x)=f(x, u(x), \nabla u(x)) .
$$

Moreover, let $I^{*}: L^{\frac{r(x)}{r(x)-1}}(\Omega) \rightarrow E^{*}$ be the adjoint operator for the embedding of $I: E \rightarrow L^{r(x)}(\Omega)$. We then define

$$
N_{f}=I^{*} \circ \bar{N}_{f}: E \rightarrow E^{*},
$$

which is well-defined by assumption $\left(f_{1}\right)$. We set

$$
\mathbb{L}(u)=\mathcal{L}(u)-N_{f}(u) .
$$

In view of Lemma 1 , it suffices to show the following: $\left(A_{1}\right) \mathbb{L}$ is bounded; $\left(A_{2}\right) \mathbb{L}$ is pseudomonotone; $\left(A_{3}\right) \mathbb{L}$ is coercive.

Verification of $\left(A_{1}\right)$ : By virtue of the growth condition $f_{1}$, we easily know that $\mathbb{L}$ maps bounded sets into bounded sets. Thus, $\left(A_{1}\right)$ is satisfied.

Verification of $\left(A_{2}\right)$ : Let $\left\{u_{n}\right\}_{n=1}^{\infty} \subset E$ be a sequence such that

$$
u_{n} \rightarrow u \text { in } E \text { and } \limsup _{n \rightarrow+\infty}\left\langle\mathbb{L}\left(u_{n}\right), u_{n}-u\right\rangle \leq 0 .
$$

Due to the compact embeddings $E \hookrightarrow L^{r(x)}(\Omega)$, we can deduct that

$$
u_{n} \rightarrow u \text { in } L^{r(x)}(\Omega) .
$$

Hence, from $\left(f_{1}\right)$, the Hölder inequality and the boundedness of $\left\{u_{n}\right\}_{n=1}^{\infty}$, it follows that 


$$
\begin{aligned}
& \quad\left|\int_{\Omega} f\left(x, u_{n}, \nabla u_{n}\right)\left(u_{n}-u\right) d x\right| \\
& \leq C \int_{\Omega} k(x)\left|u_{n}-u\right| d x+C \int_{\Omega}\left|u_{n}\right|^{r(x)-1}\left|u_{n}-u\right| d x \\
& \quad+C \int_{\Omega}\left|\nabla u_{n}\right|^{p(x) \frac{r(x)-1}{r(x)}}\left|u_{n}-u\right| d x \\
& \leq 2 C|k|_{\frac{r(x)}{r(x)-1}}\left|u_{n}-u\right|_{r(x)}+\left.\left.2 C|| u_{n}\right|^{r(x)-1}\right|_{\frac{r(x)}{r(x)-1}}\left|u_{n}-u\right|_{r(x)} \\
& \quad+\left.\left.2 C|| \nabla u_{n}\right|^{p(x) \frac{r(x)-1}{r(x)}}\right|_{\frac{r(x)}{r(x)-1}}\left|u_{n}-u\right|_{r(x)} \\
& \leq 2 C|k|_{\frac{r(x)}{r(x)-1}}\left|u_{n}-u\right|_{r(x)}+2 C\left(\left|u_{n}\right|_{r(x)}^{r^{+}-1}+\left|u_{n}\right|_{r(x)}^{r^{-}-1}\right)\left|u_{n}-u\right|_{r(x)} \\
& \quad+2 C\left(\left|\nabla u_{n}\right|_{p(x)}^{p^{+}\left(1-\frac{1}{r^{+}}\right)}+\left|\nabla u_{n}\right|_{p(x)}^{p^{-}\left(1-\frac{1}{r^{-}}\right)}\right)\left|u_{n}-u\right|_{r(x)} .
\end{aligned}
$$

This fact combined with (6) implies that

$$
\lim _{n \rightarrow+\infty} \int_{\Omega} f\left(x, u_{n}, \nabla u_{n}\right)\left(u_{n}-u\right) d x=0 .
$$

At this point, we can pass to the limit in (4) replacing $u$ with $u_{n}$ and $v$ with $u_{n}-u$. Thus, we obtain that

$$
\limsup _{n \rightarrow+\infty}\left\langle\mathcal{L}\left(u_{n}\right), u_{n}-u\right\rangle=\limsup _{n \rightarrow+\infty}\left\langle\mathbb{L}\left(u_{n}\right), u_{n}-u\right\rangle \leq 0 .
$$

Therefore, it follows that $u_{n} \rightarrow u$ because $\mathcal{L}$ is a mapping of type $\left(S_{+}\right)$. Moreover, due to the continuity of $\mathbb{L}$, we have that $\mathbb{L}\left(u_{n}\right) \rightarrow \mathbb{L}(u)$ in $E^{*}$ which proves that $\mathbb{L}$ is pseudomonotone. Therefore, $\left(A_{2}\right)$ is satisfied.

Verification of $\left(A_{3}\right)$ : To this end, we need only to show that

$$
\lim _{\|u\| \rightarrow+\infty} \frac{\langle\mathbb{L}(u), u\rangle}{\|u\|}=+\infty
$$

Indeed, for any $u \in E$ with $\|u\|>1$, it follows from $\left(f_{2}\right)$ that

$$
\begin{aligned}
\langle\mathbb{L}(u), u\rangle= & \int_{\Omega}|\nabla u|^{p(x)} d x-\int_{\Omega} f(x, u, \nabla u) u d x \\
\geq & \int_{\Omega}|\nabla u|^{p(x)} d x-b_{1} \int_{\Omega}|\nabla u|^{p(x)} d x-b_{2} \int_{\Omega}|u|^{p(x)} d x \\
& -\int_{\Omega}|w(x)| d x \\
\geq & \int_{\Omega}|\nabla u|^{p(x)} d x-b_{1} \int_{\Omega}|\nabla u|^{p(x)} d x-b_{2} \lambda_{*}^{-1} \int_{\Omega}|\nabla u|^{p(x)} d x \\
& -|w|_{1} \\
= & \left(1-b_{1}-b_{2} \lambda_{*}^{-1}\right) \int_{\Omega}|\nabla u|^{p(x)} d x-|w|_{1} .
\end{aligned}
$$

Therefore, since $p^{-}>1$ and $b_{1}+b_{2} \lambda_{*}^{-1}<1$, by using (11) and Proposition 1 , we have

$$
\langle\mathbb{L}(u), u\rangle \geq\left(1-b_{1}-b_{2} \lambda_{*}^{-1}\right)\|u\|^{p^{-}}-|w|_{1},
$$

which implies that (10) is satisfied and, hence, $\mathbb{L}$ is coercive. Thus, $\left(A_{3}\right)$ is satisfied.

Therefore, all the assumptions of Lemma 1 are satisfied; hence, there exists a $u_{0} \in E$ such that $\mathbb{L}(u)=0$, which concludes the proof of Theorem 1 .

Finally, we give the proof of Theorem 2. 
Proof of Theorem 2. Let $u_{1}, u_{2} \in E$ be two weak solutions of (1). Considering the weak formulation of $u_{1}$ and $u_{2}$, by choosing $v=u_{1}-u_{2}$ as a test function, we have

$$
\int_{\Omega}\left|\nabla u_{1}\right|^{p(x)-2} \nabla u_{1} \cdot \nabla\left(u_{1}-u_{2}\right) d x=\int_{\Omega} f\left(x, u_{1}, \nabla u_{1}\right)\left(u_{1}-u_{2}\right) d x
$$

and

$$
\int_{\Omega}\left|\nabla u_{2}\right|^{p(x)-2} \nabla u_{2} \cdot \nabla\left(u_{1}-u_{2}\right) d x=\int_{\Omega} f\left(x, u_{2}, \nabla u_{2}\right)\left(u_{1}-u_{2}\right) d x .
$$

Subtracting the above two equations, we have

$$
\begin{aligned}
& \int_{\Omega}\left(\left|\nabla u_{1}\right|^{p(x)-2} \nabla u_{1}-\left|\nabla u_{2}\right|^{p(x)-2} \nabla u_{2}\right) \cdot \nabla\left(u_{1}-u_{2}\right) d x \\
= & \int_{\Omega}\left(f\left(x, u_{1}, \nabla u_{1}\right)-f\left(x, u_{2}, \nabla u_{2}\right)\right)\left(u_{1}-u_{2}\right) d x .
\end{aligned}
$$

Moreover, since $p(x) \geq 2$ for all $x \in \bar{\Omega}$, then we have the following inequality (see [48]):

$$
\begin{array}{r}
\left(\left|\nabla u_{1}(x)\right|^{p(x)-2} \nabla u_{1}(x)-\left|\nabla u_{2}(x)\right|^{p(x)-2} \nabla u_{2}(x)\right) \cdot \nabla\left(u_{1}(x)-u_{2}(x)\right) \\
\geq\left(\frac{1}{2}\right)^{p(x)}\left|\nabla\left(u_{1}(x)-u_{2}(x)\right)\right|^{p(x)} .
\end{array}
$$

Using assumptions $\left(f_{3}\right)-\left(f_{4}\right)$ and (14) in (13), then it follows from the Young inequality that

$$
\begin{aligned}
& \left(\frac{1}{2}\right)^{p^{+}} \int_{\Omega}\left|\nabla\left(u_{1}(x)-u_{2}(x)\right)\right|^{p(x)} d x \\
\leq & \int_{\Omega}\left(\left|\nabla u_{1}\right|^{p(x)-2} \nabla u_{1}-\left|\nabla u_{2}\right|^{p(x)-2} \nabla u_{2}\right) \cdot \nabla\left(u_{1}-u_{2}\right) d x \\
= & \int_{\Omega}\left(f\left(x, u_{1}, \nabla u_{1}\right)-f\left(x, u_{2}, \nabla u_{2}\right)\right)\left(u_{1}-u_{2}\right) d x \\
= & \int_{\Omega}\left(f\left(x, u_{1}, \nabla u_{1}\right)-f\left(x, u_{2}, \nabla u_{1}\right)\right)\left(u_{1}-u_{2}\right) d x \\
& +\int_{\Omega}\left(f\left(x, u_{2}, \nabla u_{1}\right)-f\left(x, u_{2}, \nabla u_{2}\right)\right)\left(u_{1}-u_{2}\right) d x \\
\leq & b_{3} \int_{\Omega}\left|u_{1}-u_{2}\right|^{p(x)} d x+b_{4} \int_{\Omega}\left|\nabla\left(u_{1}-u_{2}\right)\right|^{p(x)-1}\left|u_{1}-u_{2}\right| d x \\
\leq & b_{3} \int_{\Omega}\left|u_{1}-u_{2}\right|^{p(x)} d x+b_{4} \int_{\Omega} \frac{\left|\nabla\left(u_{1}-u_{2}\right)\right|^{p(x)}}{\frac{p(x)}{p(x)-1}} d x \\
& +b_{4} \int_{\Omega} \frac{\left|u_{1}-u_{2}\right|^{p(x)}}{p(x)} d x \\
\leq & b_{3} \lambda_{*}^{-1} \int_{\Omega}\left|\nabla\left(u_{1}-u_{2}\right)\right|^{p(x)} d x+b_{4} \frac{p^{+}-1}{p^{-}} \int_{\Omega}\left|\nabla\left(u_{1}-u_{2}\right)\right|^{p(x)} d x \\
& +\frac{b_{4}}{p^{-}} \lambda_{*}^{-1} \int_{\Omega}\left|\nabla\left(u_{1}-u_{2}\right)\right|^{p(x)} d x \\
= & \left(\left[b_{3}+\frac{b_{4}}{p^{-}}\right] \lambda_{*}^{-1}+b_{4} \frac{p^{+}-1}{p^{-}}\right) \int_{\Omega}\left|\nabla\left(u_{1}-u_{2}\right)\right|^{p(x)} d x .
\end{aligned}
$$

Consequently, when $2^{p^{+}}\left(\left[b_{3}+\frac{b_{4}}{q^{-}}\right] \lambda_{* *}^{-1}+b_{4} \frac{q^{+}-1}{q^{-}}\right)<1$, it follows from inequality (15) that $u_{1}=u_{2}$ and so the solution of (1) is unique. The proof is complete. 


\section{Conclusions}

In this paper, we have discussed the $p(x)$-Laplacian equation with a convection term on the gradient. Due to the presence of the gradient $\nabla u$ in the term $f(x, u, \nabla u)$, problem (1) does not have a generally variational structure, so the variational methods are not applicable. In view of this difficulty, few papers pay attention to the existence of solutions to problem (1) in its general form. In order to overcome this difficulty, in this paper, we use the theory of pseudomonotone operators to obtain the existence of solutions for problem (1), formulated in the paper as Theorem 1. Furthermore, under some additional conditions of the data, the uniqueness of weak solutions is shown, formulated in the paper as Theorem 2. One example is given to show the effectiveness of our results.

Author Contributions: Conceptualization, B.-S.W. and B.G.; investigation, G.-L.H. and B.G.; methodology, G.-L.H. and B.G. All authors have read and agreed to the published version of the manuscript.

Funding: This work was supported by the National Natural Science Foundation of China (No. 11201095), the TMD integral validation test of PCS tank (No. KY10200200047), the Fundamental Research Funds for the Central Universities (No. 3072020CF2401), the postdoctoral research startup foundation of Heilongjiang (No. LBH-Q14044), and the Science Research Funds for Overseas Returned Chinese Scholars of Heilongjiang Province (No. LC201502).

Conflicts of Interest: The authors declare no conflict of interest.

\section{References}

1. Ruzicka, M. Electrorheological Fluids: Modeling and Mathematical Theory; Lecture Notes in Mathematics; Springer: Berlin, Germany, 2000; Volume 1748.

2. Rajagopal, K.R.; Ruzicka, M. Mathematical modeling of electrorheological materials. Contin. Mech. Thermodyn. 2001, 13, 59-78. [CrossRef]

3. Halsey, T.C. Electrorheological fluids. Science 1992, 258, 761-766. [CrossRef]

4. Zhikov, V.V. Averaging of functionals of the calculus of variations and elasticity theory. Math. USSR. Izv. 1987, 9, 33-66. [CrossRef]

5. Chen, Y.M.; Levine, S.; Rao, M. Variable exponent, linear growth functionals in image restoration. SIAM J. Appl. Math. 2006, 66, 1383-1406. [CrossRef]

6. Tiirola, J. Image decompositions using spaces of variable smoothness and integrability. SIAM J. Imaging Sci. 2014, 7, 1558-1587. [CrossRef]

7. Zhang, D.Z.; Shi, K.H.; Guo, Z.C.; Wu, B.Y. A class of elliptic systems with discontinuous variable exponents and data for image denoising. Nonlinear Anal. Real World Appl. 2019, 50, 448-468. [CrossRef]

8. Cekic, B.; Kalinin, A.V.; Mashiyev, R.; Avci, M. $L^{p(x)}(\Omega)$-estimates of vector fields and some applications to magnetostatics problems. J. Math. Anal. Appl. 2012, 389, 838-851. [CrossRef]

9. Kefi, K.; Radulescu, V.D. On a $p(x)$-biharmonic problem with singular weights, Z. Angew. Math. Phys. 2017, 68, 1-13. [CrossRef]

10. Saoudi, K. The fibering map approach to a $p(x)$-Laplacian equation with singular nonlinearities and nonlinear Neumann boundary conditions. Rocky Mt. J. Math. 2018, 48, 927-946. [CrossRef]

11. Kefi, K.; Saoudi, K. On the existence of a weak solution for some singular $p(x)$-biharmonic equation with Navier boundary conditions. Adv. Nonlinear Anal. 2019, 8, 1171-1183. [CrossRef]

12. Xie, W.L.; Chen, H.B. Existence and multiplicity of solutions for $p(x)$-Laplacian equations in $\mathbb{R}^{N}$. Math. Nachr. 2018, 291, 2476-2488. [CrossRef]

13. Nhan, L.C.; Chuong, Q.V.; Truong, L.X. Potential well method for $p(x)$-Laplacian equations with variable exponent sources. Nonlinear Anal. Real World Appl. 2020, 56, 103155. [CrossRef]

14. Zhang, X. A minimization problem with variable growth on Nehari manifold. Monatsh. Math. 2016, 181, 485-500. [CrossRef]

15. Zhang, Q.H.; Motreanu, D. Existence and blow-up rate of large solutions of $p(x)$-Laplacian equations with large perturbation and gradient terms. Adv. Differ. Equ. 2016, 21, 699-734.

16. Yucedag, Z. Solutions of nonlinear problems involving $p(x)$-Laplacian operator. Adv. Nonlinear Anal. 2015, 4, 285-293. [CrossRef] 
17. Yin, J.X.; Li, J.K.; Ke, Y.Y. Existence of positive solutions for the $p(x)$-Laplacian equation. Rocky Mt. J. Math. 2012, 42, 1675-1758. [CrossRef]

18. Hammou, M.A.; Azroul, E.; Lahmi, B. Existence of solutions for $p(x)$-Laplacian Dirichlet problem by topological degree. Bull. Transilv. Univ. Bras. Ser. III 2018, 11, 29-38.

19. Ayazoglu, R.; Ekincioglu, I. Electrorheological fluids equations involving variable exponent with dependence on the gradient via mountain pass techniques. Numer. Funct. Anal. Optim. 2016, 37, 1144-1157.

20. Ge, B.; Wang, L.Y. Infinitely many solutions for a class of superlinear problems involving variable exponents. Adv. Differ. Equ. 2020, 25, 191-212.

21. Ge, B.; Lv, D.J. Superlinear elliptic equations with variable exponent via perturbation method. Acta Appl. Math. 2020, 166, 85-109. [CrossRef]

22. Ge, B. Vicentiu D. Radulescu, Infinitely many solutions for a non-homogeneous differential inclusion with lack of compactness. Adv. Nonlinear Stud. 2019, 19, 625-637. [CrossRef]

23. Heidarkhani, S.; Afrouzi, G.A.; Moradi, S.; Caristi, G.; Ge, B. Existence of one weak solution for $p(x)$-biharmonic equations with Navier boundary conditions. Z. Angew. Math. Phys. 2016, 67, 1-13. [CrossRef]

24. Ge, B.; Zhou, Q.M.; Wu, Y.H. Eigenvalues of the $p(x)$-biharmonic operator with indefinite weight. Z. Angew. Math. Phys. 2015, 66, 1007-1021. [CrossRef]

25. Fan, X.L. Existence and uniqueness for the $p(x)$-Laplacian Dirichlet problems. Math. Nachr. 2011, 284, 1435-1445. [CrossRef]

26. Fan, X.L. Global $C^{1, \alpha}$ regularity for variable exponent elliptic equations in divergence form. J. Differ. Equ. 2007, 235, 397-417. [CrossRef]

27. Ding, M.Y.; Zhang, C.; Zhou, S.L. On optimal $C^{1, \alpha}$ estimates for $p(x)$-Laplace type equations. Nonlinear Anal. 2020, 200, 112030. [CrossRef]

28. Ok, J. Harnack inequality for a class of functionals with non-standard growth via De Giorgi's method. Adv. Nonlinear Anal. 2018, 7, 167-182. [CrossRef]

29. Yao, F.P. Local Holder estimates for non-uniformly variable exponent elliptic equations in divergence form. Proc. R. Soc. Edinb. Sect. A 2018, 148, 211-224. [CrossRef]

30. Byun, S.S.; Ok, J.; Park, J. Regularity estimates for quasilinear elliptic equations with variable growth involving measure data. Ann. Inst. Henri Poincare Anal. Non Lineaire 2017, 34, 1639-1667. [CrossRef]

31. Byun, S.S.; Ok, J.; Ryu, S. Global gradient estimates for elliptic equations of $p(x)$-Laplacian type with BMO nonlinearity. J. Reine Angew. Math. 2016, 715, 1-38. [CrossRef]

32. Byun, S.S.; Lee, M.; Ok, J. $W^{2, p(x)}$-regularity for elliptic equations in nondivergence form with BMO coefficients. Math. Ann. 2015, 363, 1023-1052. [CrossRef]

33. Baroni, P.; Habermann, J. Elliptic interpolation estimates for non-standard growth operators. Ann. Acad. Sci. Fenn. Math. 2014, 39, 119-162. [CrossRef]

34. Giannetti, F.; di Napoli Passarelli, A. Regularity results for a new class of functionals with non-standard growth conditions. J. Differ. Equ. 2013, 254, 1280-1305. [CrossRef]

35. Harjulehto, P.; Hasto, P.; Latvala, V.; Toivanen, O. The strong minimum principle for quasisuperminimizers of non-standard growth. Ann. Inst. Henri Poincare Anal. Non Lineaire 2011, 28, 731-742. [CrossRef]

36. Adamowicz, T.; Hasto, P. Harnack's inequality and the strong $p(\cdot)$-Laplacian. J. Differ. Equ. 2011, 250, 1631-1649. [CrossRef]

37. Montenegro, M.; Montenegro, M. Existence and nonexistence of solutions for quasilinear elliptic equations. J. Math. Anal. Appl. 2000, 245, 303-316. [CrossRef]

38. Ruiz, D. A priori estimates and existence of positive solutions for strongly nonlinear problems. J. Differ. Equ. 2004, 199, 96-114. [CrossRef]

39. Zou, H.H. A priori estimates and existence for quasi-linear elliptic equations. Calc. Var. Partial Differ. Equ. 2008, 33, 417-437. [CrossRef]

40. Figueiredo, G.M. Quasilinear equations with dependence on the gradient via mountain pass techniques in $\mathbb{R}^{N}$. Appl. Math. Comput. 2008, 203, 14-18.

41. Faraci, F.; Motreanu, D.; Puglisi, D. Positive solutions of quasi-linear elliptic equations with dependence on the gradient. Calc. Var. Partial Differ. Equ. 2015, 54, 525-538. [CrossRef]

42. Fan, X.L.; Zhang, Q.H.; Zhao, D. Eigenvalues of $p(x)$-Laplacian Dirichlet problem. J. Math. Anal. Appl. 2005, 302, 306-317. [CrossRef] 
43. Fan, X.L.; Zhang, Q.H. Existence of solutions for $p(x)$-Laplacian Dirichlet problems. Nonlinear Anal. 2003, 52, 1843-1852. [CrossRef]

44. Fan, X.L.; Zhao, D. On the spaces $L^{p(x)}(\Omega)$ and $W^{m, p(x)}(\Omega)$. J. Math. Anal. Appl. 2001, 263, $424-446$. [CrossRef]

45. Diening, L.; Harjuletho, P.; Hasto, P.; Ruzicka, M. Lebesgue and Sobolev Spaces with Variable Exponents; Springer: Berlin, Germany, 2011.

46. Kovacik, O.; Rakosnik, J. On spaces $L^{p(x)}$ and $W^{1, p(x)}$. Czechoslov. Math. J. 1991, 41, 592-618.

47. Carl, S.; Le, V.K.; Motreanu, D. Nonsmooth Variational Problems and Their Inequalities; Springer: New York, NY, USA, 2007.

48. Kichenassamy, S.; Veron, L. Singular solutions of the $p$-Laplace equation. Math. Ann. 1985, 275, 599-615. [CrossRef]

Publisher's Note: MDPI stays neutral with regard to jurisdictional claims in published maps and institutional affiliations.

(C) 2020 by the authors. Licensee MDPI, Basel, Switzerland. This article is an open access article distributed under the terms and conditions of the Creative Commons Attribution (CC BY) license (http:/ / creativecommons.org/licenses/by/4.0/). 